\title{
Évaluation du risque de cancer du sein héréditaire : BRCA1 et BRCA2
}

par Kendra-Ann I. Seenandan-Sookdeo et Jo-Ann V. Sawatzky

\section{Abrégé}

La récente prolifération des travaux de recherche génétique sur le cancer du sein héréditaire a rehaussé notre compréhension des influences génétiques sur la santé et sur les maladies. Ces progrès scientifiques ont des répercussions importantes pour les infirmières cliniciennes en oncologie et les infirmières en pratique avancée. Les auteures de cet article utilisent le Human Response to Illness Model [modèle de réponse humaine à la maladie] (Mitchell, Gallucci \& Fought, 1991) afin d'acquérir une compréhension approfondie du cancer du sein concernant la réponse humaine aux mutations génétiques. Il soulignera donc la documentation portant sur les perspectives physiologiques, pathophysiologiques, comportementales et expérientielles liées au cancer du sein dû à une mutation de BRCA. Ces résultats fondés sur la recherche procureront aux infirmières en oncologie les compétences leur permettant d'élaborer un plan de soins holistiques pour les femmes ayant un risque génétique-actuel ou éventuel-de cancer du sein.

\section{Évaluation du risque de cancer du sein héréditaire : BRCA1 et BRCA2}

Le cancer du sein touche la vie de bien des individus et familles à l'échelle mondiale. Au Canada, le cancer du sein est le type de cancer le plus fréquemment diagnostiqué chez les femmes et il se place au deuxième rang en termes de taux de mortalité chez les femmes, derrière le cancer du poumon. En 2009, on estime que 22900 Canadiennes recevront un diagnostic positif de cancer du sein, ce qui se traduira par 5400 décès (Société canadienne du cancer, 2009). Une lourde histoire familiale de cancer du sein rehausse le profil de risque de la personne relativement à la maladie. De plus, une mutation génique positive du cancer du sein exercera une incidence additionnelle sur le profil de risque. Le cancer du sein héréditaire représente entre 5 et $10 \%$ de tous les diagnostics de cancer du sein, dont environ 3 à $7 \%$ concernent une mutation positive des gènes BRCA1 ou BRCA2 (Carroll, Cremin, Allanson, Blaine, Dorman, Gibbons et coll., 2008). Les personnes porteuses de ces gènes ont entre 50 et $87 \%$ de chances de développer un cancer du sein d'ici l'âge de soixante-dix ans (Berliner \& Fay, 2007).

Cet article met à profit le Human Response to Illness (HRTI) Model [modèle de Réponse humaine à la maladie] (Mitchell et coll., 1991) afin d'orienter un examen structuré de la réponse humaine au cancer du sein héréditaire-éventuel ou réel-lié aux gènes BRCA, en présentant les quatre perspectives interdépendantes impliquées dans la complexe réponse humaine à la maladie. Ces quatre perspectives comprennent des aspects physiologiques, pathophysiologiques, comportementaux et expérientiels. Des résultats de recherche seront utilisés afin de dégager une compréhension détaillée du cancer du sein héréditaire, des outils d'évaluation du risque, les enjeux psychologiques et les implications pour les infirmières en oncologie. Cet examen fournira donc aux infirmières cliniciennes les connaissances nécessaires au déclenchement d'une évaluation des antécédents de cancer du sein fondée sur des données probantes, à l'éducation de la clientèle, à des consultations axées sur le soutien et à l'aiguillage vers les services de génétique requis.

\section{Perspective physiologique}

Selon Mitchell et ses collègues (1991), les principes des réponses régulatrices d'ordre physiologique proviennent d'une compréhension du fonctionnement biologique normal de l'organisme; il faut donc qu'une discussion sur le cancer du sein héréditaire débute par les rudiments de la biologie cellulaire et du rôle de BRCAl et de BRCA2 dans les activités complexes ayant lieu au niveau du noyau cellulaire (Loescher \& Whitesell, 2006). La littérature contient de multiples preuves que le cancer est une maladie génétique due à des mutations de gènes. Toute mutation qui provoque une accélération du rythme de division cellulaire ou un ralentissement du rythme normal de mort cellulaire, peut contribuer à la croissance désordonnée de cellules cancéreuses (Loescher et coll., 2006). Les cellules somatiques de l'organisme (c.-à-d. toutes les cellules de l'organisme à l'exception des cellules sexuelles) renferment un noyau cellulaire lequel contient des chromosomes comprenant les compactes unités fonctionnelles de l'hérédité mieux connues sous le nom de gènes. Ces derniers se composent de séquences d'acide désoxyribonucléique $(\mathrm{ADN})$. Celui-ci assure le codage de près de 30000 gènes se trouvant dans les 23 paires de chromosomes des cellules humaines (Loescher et coll., 2006). Parmi ces 23 paires de chromosomes, un chromosome de chaque paire est fourni aux descendants en provenance du patrimoine maternel et du patrimoine paternel. Un descendant a donc $50 \%$ de chances d'hériter d'une mutation autosomique dominante.

Bien qu'il y ait deux catégories de gènes qui jouent un rôle clé dans la régulation cellulaire (c.-à-d. les gènes suppresseurs de tumeurs et les proto-oncogènes), cet examen va se concentrer sur deux gènes suppresseurs de tumeurs, BRCA1 et BRCA2. Ceux-ci sont impliqués dans la transcription de l'ADN et assurent la régulation des activités cellulaires en bloquant les protéines favorisant la croissance afin de permettre la réparation des cellules endom-

\section{Au sujet des auteures}

Kendra-Ann I. Seenandan-Sookdeo, B.Sc.inf., inf., Étudiante diplômée, CancerCare Manitoba/Manitoba Institute of Cell Biology-Breast Tumour Bank, Office régional de la santé de Winnipeg/Centre des sciences de la santé-Hereditary Breast \& Ovarian Cancer Clinic, Infirmière à la Clinique du sein, Office régional de la santé de Winnipeg-Centre de santé du sein. Courriel : KendraAnn.Seenandan@cancercare.mb.ca

Jo-Ann V. Sawatzky, inf., Ph.D., Professeure agrégée, Faculté des Sciences infirmières, Université du Manitoba.

Les auteures reconnaissent avec gratitude la participation des personnes suivantes à la révision d'une version préliminaire de cet article :

$D^{r}$ Leigh Murphy, directeur par intérim et scientifique principal du CancerCare Manitoba Institute of Cell Biology

Kim Serfas, conseillère en génétique, Office régional de la santé de Winnipeg : Centre des sciences de la santé-Hereditary Breast and Ovarian Clinic.

Réalisé grâce à l'appui financier suivant à la première auteure : Bourse d'étude de la Fondation canadienne de la recherche sur les services de santé/Instituts de recherche en santé du Canada (chaire Élaboration de pratiques infirmières fondées sur les données probantes en oncologie, soins palliatifs et prévention $d u$ cancer) détenue par la $D^{r e}$ Lesley Degner, Faculté des Sciences infirmières, Université du Manitoba.

" Je dédie cet article à ma grand-mère Winnifred Ramsay. " -Kendra-Ann I. Seenandan 
magées (Yoshida \& Miki, 2004). Ces deux gènes participent également à la régulation des signaux relatifs à l'apoptose, c'est-à-dire la mort cellulaire programmée (Loescher et coll., 2006). Nardo et Foulkes (2004) soulignent que le génome humain doit sa préservation à l'implication, à divers degrés, des protéines BRCA dans de multiples étapes du cycle cellulaire, dont les suivantes : points de contrôle du cycle cellulaire, ubiquitination, remodelage de la chromatine et enfin, recombinaison homologue. Ces séries d'évènements font partie intégrante du type de division cellulaire appelé mitose. Les points de contrôle empêchent les cellules de passer à la phase suivante avant qu'elles ne soient prêtes pour la division cellulaire complète. L'ubiquitination est un processus du cycle cellulaire qui marque les protéines en voie de dégradation. Le remodelage de la chromatine assure la réparation des cassures double brin d'ADN, et la recombinaison homologue est le mécanisme qui gère les échanges de fragments d'ADN double brin (Narod et coll., 2004). Les protéines BRCA jouent donc un rôle capital de contrôle en maintenant un certain nombre de mécanismes cellulaires.

\section{Perspective pathophysiologique}

D’après Mitchell et ses collègues (1991), les réponses pathophysiologiques aboutissent à une décompensation biologique; ainsi, une altération d'un gène BRCA accroîtrait chez l'individu concerné les risques de problèmes de santé. Une mutation dommageable de l'un ou l'autre des gènes BRCA augmente la susceptibilité d'une personne au cancer du sein et de l'ovaire et à d'autres types de cancers (Berliner et coll., 2007). Alors que la majorité des cancers du sein sont sporadiques et dus à des mutations somatiques, la susceptibilité au cancer héréditaire est liée aux mutations des cellules germinales (Fackenthal \& Olopade, 2007).

Chapman (2007) définit les mutations germinales comme étant des altérations génétiques héréditaires qui se trouvent dans les ovules ou le sperme et qui sont transmises aux descendants. Une revue de la littérature réalisée par Narod et ses collaborateurs (2004) continue d'appuyer la théorie du double événement mutationnel relatif au cancer du sein héréditaire. Selon cette théorie, le cancer peut survenir lorsqu'un individu ayant hérité d'une mutation du gène BRCA en provenance d'un de ses parents subit une mutation somatique du gène BRCA hérité de l'autre parent. Dans un tiers de ces mutations, la signification clinique n'est pas spécifiée; par conséquent, certaines d'entre elles peuvent être des variantes normales non accompagnées de conséquences pathologiques puisque la mutation aboutit malgré tout à la création d'une protéine fonctionnelle, tandis que d'autres peuvent prédisposer l'individu au cancer étant donné que la protéine ainsi obtenue est non fonctionnelle (Loescher et coll., 2006).

Les gènes BRCA défectueux ont des conséquences potentiellement négatives sur la transcription de l'ADN, la régulation du cycle cellulaire, la réparation de l'ADN, les points de contrôle du cycle cellulaire, l'ubiquitination et le remodelage de la chromatine. Selon Yoshida et ses collègues (2004), les protéines BRCA altérées peuvent nuire aux relations protéiques essentielles. Ceci fait que des changements au niveau des gènes BRCA peuvent contribuer au développement de tumeurs dans le cadre du processus dénommé tumorigenèse (Yoshida et coll., 2004).

\section{Perspective comportementale}

Dans le modèle HRTI, les réponses comportementales sont décrites sous forme de comportements observables et mesurables lesquels peuvent indiquer la présence d'un signe ou symptôme sous-jacent (Mitchell et coll., 1991). Le modèle suggère également que des facteurs environnementaux ainsi que des facteurs non modifiables (p. ex. la constitution génétique) et des facteurs modifiables (p. ex. le mode de vie) entretiennent des interactions avec les quatre perspectives de la réponse humaine à la maladie et influencent la réponse de l'individu face à la maladie. Des outils d'évalua- tion du risque permettent de mesurer la présence éventuelle chez un individu d'un risque héréditaire non modifiable de mutation BRCA (Mitchell et coll., 1991). Dans le milieu des soins primaires, l'outil de mesure fondamental est une évaluation détaillée des antécédents oncogénétiques qui prend en compte les problèmes de santé personnels et familiaux dans la détermination de l'admissibilité à la consultation génétique (Willey \& Cocilovo, 2007). Le modèle de Gail est d'un usage limité dans ce contexte puisqu'il entraîne une sous-estimation du cancer du sein chez les personnes qui ont une lourde histoire familiale en matière de cancer du sein et de l'ovaire, mais qui n'ont pas de parents au premier degré touchés par la maladie (Willey et coll., 2007).

Les outils d'évaluation génétique jouent un rôle essentiel dans l'identification des individus et de leurs proches à risque élevé de développer un cancer. Il est donc nécessaire que les infirmières déterminent si un individu donné répond aux exigences de la référence vers un conseiller en hérédité auprès duquel il subira un éventuel dépistage génétique. Cette démarche débute par une méticuleuse évaluation infirmière de l'état de santé portant une attention particulière aux antécédents de la cliente en santé du sein et à l'histoire familiale en matière de cancer.

\section{Évaluation des antécédents personnels de santé du sein}

Les éléments clés d'une anamnèse personnelle comprennent des facteurs de risque non modifiables tels que des antécédents de tumeurs ou de problèmes bénins des seins, le sexe, l'âge et une histoire menstruelle et reproductive. Les facteurs modifiables incluent entre autres l'utilisation de contraceptifs oraux (CO), l'hormonothérapie substitutive (HTS), le tabagisme, la consommation d'alcool, l'obésité et l'activité physique. L'histoire de santé du sein évalue également des facteurs environnementaux comme l'exposition aux rayonnements ou à des produits chimiques carcinogènes connus.

Des antécédents d'anomalies malignes ou bénignes ont une incidence sur le profil de risque en matière de cancer du sein. Un déclencheur automatique de la référence en génétique est l'apparition précoce du cancer du sein (c.-à-d. avant 50 ans) laquelle multiplie par cinq le risque de cancer tandis que des antécédents personnels de cancer de l'endomètre ou de l'ovaire multiplient ce risque par deux (Willey et coll., 2007). Une combinaison de l'apparition précoce du cancer du sein ou de l'ovaire (à n'importe quel âge pour ce dernier) et/ou d'un cancer bilatéral (ou multifocal) rehausse également le profil de risque (Petrucelli, Daly, Culver \& Feldman, 2007). Plusieurs études ont démontré que des profils élevés de risque de carcinome mammaire comprennent des résultats histologiques bénins au niveau du sein tels que le carcinome lobulaire in situ, l'hyperplasie lobulaire atypique, l'hyperplasie canalaire atypique et l'atypie (Brown, Moglia \& Grumet, 2007).

Les hormones ont une incidence sur la croissance cellulaire; cela signifie que les changements de niveau d'œstrogène et de progestérone dans le tissu mammaire de la femme augmentent la susceptibilité au cancer du sein chez elle par rapport à l'homme (Willey et coll., 2007). Pour ce qui est de l'âge, c'est avant 40 ans que le cancer du sein apparaît généralement chez les femmes porteuses de la mutation BRCA1 tandis que chez les porteuses du BRCA2, la fréquence du cancer augmente après l'âge de 50 ans (Nkondjock \& Ghadirian, 2004).

Rosen, Fan et Isaacs (2005) avancent qu'en ce qui concerne les femmes porteuses d'une mutation BRCA, le fait d'avoir mené une grossesse à terme à un jeune âge, une parité élevée et une ménarche tardive exercent un effet protecteur contre le cancer du sein. Dans une étude plus récente, Kotsopoulos et ses collègues (2007) affirment que les influences protectrices du jeune âge lors de la première grossesse menée à terme ne sont pas constatées chez les femmes porteuses d'une mutation BRCA et que bien que la multiparité confère de la protection aux porteuses de la mutation germinale 
BRCA1, cet effet protecteur ne s'applique pas aux porteuses de BRCA2 chez qui l'étiologie sous-jacente n'est toujours pas entièrement comprise.

Quoique l'emploi d'hormones exogènes chez les personnes porteuses d'une mutation BRCA ait fait l'objet d'études approfondies au fil des ans, les résultats sont peu cohérents. Le National Hereditary Cancer Task Force (NHCTF) lance un avertissement concernant la recommandation d'utiliser les CO chez les femmes que l'on sait porteuses d'une mutation BRCA âgées de 35 ans et plus du fait de l'accroissement potentiel de leur risque de cancer du sein. Des études sur la HTS et sur les porteuses d'une mutation de BRCA n'ont été réalisées qu'auprès de femmes ayant subi une salpingoovariectomie bilatérale (SOB) prophylactique. Le NHCTF appuie l'utilisation de la HTS (œstrogènes uniquement) pour la prise en charge à court terme des symptômes de la ménopause, si les traitements non hormonaux n'ont pas réussi à les soulager (Horsman, 2007).

Les résultats concernant le BRCA et le tabagisme ne sont guère plus cohérents. Par exemple, Cuningham (2008) a récemment fait état d'une hausse linéaire des taux de cancer du sein chez les porteuses des mutations qui fumaient des cigarettes; ce résultat contredisait les rapports signalant une absence d'association entre ces variables. Les résultats de McGuire et collaborateurs (2006) viennent appuyer les résultats de recherche précédents démontrant un risque accru du fait de la consommation d'alcool chez les femmes porteuses d'une mutation BRCA de moins de 50 ans. Les travaux de recherche rapportent également de façon constante un retard dans l'apparition du cancer du sein chez les porteuses de mutation qui se livrent à des activités physiques et maintiennent un poids corporel sain (Roncker, Erdmann \& Land, 2005). Les résultats de recherche liés au risque associé de cancer du sein chez les femmes porteuses d'une mutation BRCA à la suite de l'exposition à des rayonnements ionisants ne sont pas concluants; toutefois, les écrits rapportent un risque accru chez toute personne ayant subi une radio-exposition avant l'âge de 30 ans (Willey et coll., 2007).

\section{Évaluation de l'histoire oncologique familiale}

Une évaluation de la santé du sein incorpore les antécédents de santé familiaux. Idéalement, cet examen des antécédents porte sur trois générations de chaque côté de la famille, en prêtant attention au nombre d'individus touchés par la maladie par rapport à celui des individus non touchés, aux types de cancers, à l'âge au moment du diagnostic, à la présence du cancer dans des générations successives et au degré de parenté entre tous les individus concernés. En outre, il convient d'obtenir des renseignements sur le sexe, l'appartenance ethnique, le schéma thérapeutique de la prise en charge du cancer et l'état de santé actuel (p. ex. encore en vie et en bonne santé ou date du décès) (Barse, 2006). Petrucelli et ses collègues (2007) recommandent que les évaluations du risque examinent également l'histoire familiale relative à plusieurs autres types de cancers et de syndromes de cancer associés à un risque accru de cancer du sein (voir le tableau 1).

\section{Dépistage génétique}

Le dépistage génétique est la méthode par excellence d'évaluation de la susceptibilité à une mutation de gène BRCA. Ce processus commence par une consultation pré-test de dépistage et par l'obtention du consentement éclairé de la personne identifiée comme présentant un risque élevé de mutation. Une cote d'évaluation du risque indiquant > 10 \% de probabilité de prédisposition à une mutation satisfait à la majorité des normes régissant la réalisation du test (Carter, 2001). Les normes régissant le dépistage génétique subissent également l'influence du raisonnement clinique fondé sur les facteurs de risque personnels et familiaux, de l'impact des résultats du test génétique sur les plans de gestion du traitement (c.-à-d. options de chirurgie par opposition à celles de chimioprévention) ainsi que les inquiétudes et les préférences connues de la cliente
(Berliner et coll., 2007). Les membres de la famille d'une personne ayant obtenu un résultat positif au test de dépistage du BRCA peuvent également se prévaloir des tests génétiques prédictifs (Ensenauer, Michels \& Reinke, 2005).

Ce test comprend habituellement l'extraction de l'ADN d'un échantillon de sang périphérique et la réalisation d'un séquençage d'ADN complet et/ou une analyse de mutations ciblées (Petrucelli et coll., 2007). On constate des variations de l'exactitude parmi les laboratoires de dépistage; ainsi, la sensibilité des séquençages complets s'étend de 80 à $95 \%$ alors que l'analyse de mutations ciblées s'avère exacte dans approximativement 99 \% des cas (Sifri, Gangadharappa \& Acheson, 2004). Brown et ses collègues (2007) résument les résultats du test comme étant positifs, négatifs ou une variante de signification incertaine. Puisque les méthodes de test ne sont pas sensibles à $100 \%$, il est essentiel d'interpréter les résultats négatifs dans le contexte clinique du cas particulier et de l'évaluation de l'histoire familiale (Ensenauer et coll., 2005). Des résultats négatifs peuvent s'expliquer de plusieurs façons possibles, notamment les suivantes : il peut s'agir d'un cancer sporadique ou il est possible que la cliente soit en réalité porteuse d'une mutation BRCA qui n'a pas été détectée lors de l'analyse ou encore qu'elle soit porteuse d'une mutation au niveau d'un autre gène de susceptibilité au cancer (Evans, Skrzynia, Susswein \& Harlan, 2005). Sinon, il est possible que le rapport signale une variante de signification clinique incertaine, ce qui veut dire qu'un résultat de test indéterminé indique des changements détectables dans la séquence d'ADN mais qu'il n'y a aucune indication nette d'une incidence quelconque sur le fonctionnement des gènes BRCA (Brown et coll., 2007). Par contre, un résultat positif confirme la présence d'une mutation au sein de la séquence génétique laquelle augmente le risque de développer un cancer du sein; toutefois, il n'indique pas nécessairement l'apparition future d'un cancer du sein. Il en ressort que l'interprétation des résultats du test et leur divulgation sont des tâches complexes et délicates; les discussions en la matière sont donc lancées par des généticiens médicaux, des conseillères en génétique et les infirmières en pratique avancée ayant reçu une formation spécialisée en génétique.

\section{Perspective expérientielle}

Mitchell et ses collaborateurs (1991) décrivent les réponses expérientielles comme étant le compte rendu par l'individu de l'examen de ses pensées et émotions personnelles, lesquelles sont influencées par ses connaissances relatives à l'expérience et de sa signification particulière. La signification culturelle et sociale de l'expérience exerce également une influence. Dans le cadre d'une récente recension tirée de la Cochrane Database of Systematic Reviews, Sivell, Iredale, Gray \& Coles (2007) constatent que les effets psychologiques de la consultation et du dépistage génétiques n'ont pas fait l'objet d'une étude approfondie dans la littérature. Toutefois, d'après les rapports existants, les clientes qui se tournent vers le dépistage génétique sont soit des femmes en bonne santé qui courent un risque accru de cancer du sein soit des femmes qui ont reçu un diagnostic de cancer du sein. Ce qui les motive à subir le test est de clarifier leurs propres perceptions vis-à-vis de leur risque élevé de cancer afin de prendre des décisions éclairées sur les stratégies de prévention et/ou les options de prise en charge médicale. Alors que les femmes ont tendance à se prévaloir du test de dépistage afin de minimiser le risque familial, elles rapportent également des préoccupations relatives à des questions d'assurance maladie et de discrimination d'emploi (Phelps, Wood, Bennett, Brian \& Gray, 2007).

Phelps et ses collaborateurs (2007) ont évalué des questionnaires remplis par des femmes ayant été référées vers la consultation génétique pour cancer du sein et ayant choisi de participer à un essai d'intervention psychologique $(\mathrm{N}=97)$. Leur analyse a révélé que les femmes se souciaient de la manière dont elles allaient s'adapter s'il s'avérait qu'elles présentaient un risque accru de cancer et de la 
manière dont elles transmettraient cette information à leur famille. De plus, ces mêmes auteurs ont découvert que l'ajustement aux résultats relatifs au risque dépendait de la perception des individus du degré de correspondance entre la démarche d'évaluation du risque et leurs propres attentes. Les résultats des études menées par Schlich-Bakker, ten Kroode \& Ausems (2006) et par Smith et ses collègues (2008) étaient similaires à ceux d'autres études qui appuient l'hypothèse selon laquelle la démarche du dépistage génétique n'entraîne pas de détresse psychologique à long terme. Smith et ses collaborateurs (2008) remarquaient qu'il n'existait aucune différence significative entre les niveaux de détresse, de perception de risque et de qualité de vie chez les femmes fréquentant une clinique de dépistage génétique. Ils ont constaté que les femmes qui refusaient le dépistage génétique et celles qui recevaient un résultat positif au test rapportaient une détresse accrue à trois mois par rapport à leurs scores de base pour la détresse globale, en fonction du Global Severity Index (indice de sévérité globale). Il est intéressant de remarquer que chez les femmes ayant obtenu un résultat positif de BRCA, les scores de détresse atteignaient leur apogée trois mois après la divulgation des résultats et revenaient à la normale aux six mois. L'étude de Schwartz, Peshkin, Hughes, Main, Isaacs et Lerman (2002) effectuée en milieu clinique confirmait ce non-accroissement de la détresse ou de risque perçu chez les femmes aux six mois après la divulgation de résultats positifs de mutation BRCA. En outre, les résultats de Schwartz et de ses collègues (2002) n'indiquaient aucun accroissement de la détresse ou du risque perçu parmi la parenté de la proposante (c.-à-d. le premier membre de la famille à subir le test) ayant obtenu des résultats positifs de mutation délétère de BRCA.

\section{Implications pour les soins infirmiers}

Le modèle HRTI fournit aux cliniciens un cadre complet pour évaluer les besoins individuels à partir des perspectives physiologique, pathophysiologique, comportementale et expérientielle. L'examen de la documentation basée sur ces perspectives éclaire la pratique clinique, l'éducation et la recherche. Le rôle de soutien de l'infirmière clinicienne en oncologie auprès des clientes tra-

Tableau 1. Indicateurs clés d'une susceptibilité héréditaire accrue aux mutations BRCA1 et BRCA2

\section{Antécédents personnels}

Apparition à un âge précoce $(<50$ ans)

Cancer bilatéral touchant des organes pairs

Cancer du sein masculin

Tumeurs multiples chez un même individu

Plusieurs générations successivement touchées :

Cancer chez au moins deux parents du premier ou deuxième degré

Apparition à un âge précoce $(<50$ ans $)$

Cancer du sein masculin

\section{Types de cancers}

Sein, ovaire, trompes de Fallope, prostate, pancréas, larynx, œsophage, estomac, vésicule biliaire, canal cholédoque,

système hématopoïétique, mélanomes

\section{Syndromes de cancer}

Syndrome de Li-Fraumeni, syndrome de Cowden, syndrome de Peutz-Jegher, cancer gastrique diffus héréditaire, ataxie-télangiectasie, syndrome de Bloom, syndrome de Werner, xeroderma pigmentosum

\section{Appartenance ethnique}

Personnes de souche juive ashkénaze, hollandaise, islandaise

Adapté de Petrucelli et coll. (2007) versant l'épreuve du dépistage génétique comprend de multiples facettes et englobe des interactions pré- et post-consultation génétique.

Des outils de mesure tels que les formulaires d'évaluation de l'histoire génétique jouent un rôle critique dans l'identification des personnes les plus susceptibles de tirer profit d'une consultation génétique (Barse, 2006). Les infirmières cliniciennes occupent une position privilégiée pour informer les clientes sur les services de dépistage génétique et pour lancer les discussions relatives à la démarche. Ces discussions préparatoires doivent aborder les rôles des autres membres de l'équipe (c.-à-d. les généticiens et les conseillères en génétique), les avantages et les limites du dépistage génétique, la durée de la démarche de dépistage ainsi que l'ensemble des résultats plausibles du test. Il est recommandé aux infirmières cliniciennes de poursuivre le dialogue avec les clientes concernant les besoins et attentes dégagés lors de la démarche génétique. De plus, il importe d'offrir des consultations de soutien et un enseignement personnalisé adapté à la décision prise par la cliente et à ses besoins particuliers. Les infirmières ne doivent pas oublier la possibilité d'une référence en oncologie psychosociale tout au long de la démarche, les besoins étant les plus forts environ trois mois après la consultation génétique initiale (Smith et coll., 2008). Une fois que les clientes ont reçu les résultats de leur test génétique, les infirmières en oncologie œuvrant dans les cliniques de santé du sein peuvent renforcer l'information ainsi que les enseignements concernant la santé, la surveillance et les options de prise en charge (Ensenauer et coll., 2005). Par conséquent, il est impératif que les infirmières qui travaillent dans ce domaine spécialisé mâ̂trisent fort bien l'interprétation des résultats des tests génétiques. Un résultat vrai négatif indique que les risques de cancer du sein de la personne concernée sont comparables à ceux de l'ensemble de la population (Horsman, 2007). L'enseignement infirmier dispensé à ces clientes doit inclure des interventions de dépistage du cancer du sein adaptées à l'âge, des stratégies de réduction des facteurs modifiables et de l'information sur les essais cliniques en matière de prévention. Lorsqu'elle se produit dans le contexte clinique où le risque va de modéré à élevé, la découverte d'une variante de signification clinique incertaine exige des discussions en collaboration entre la cliente, d'une part, et les spécialistes de la génétique, le personnel infirmier et divers membres de l'équipe d'oncologie médicale, d'autre part.

L'enseignement destiné aux clientes ayant pris connaissance des résultats positifs de leur test doit comprendre des consultations et un enseignement personnalisés concernant le risque de développer un cancer du sein ou d'autres cancers d'origine génétique et ce, en fonction de l'âge, les stratégies de surveillance, les options chirurgicales et médicales de prévention et de traitement, les essais cliniques éventuels, les conséquences pour les membres de la famille et les options relatives à la divulgation de l'information familiale ainsi que les consultations axées sur le soutien avec les références multidisciplinaires qui s'imposent (Sivell et coll., 2007; Phelps et coll., 2007).

Les progrès du dépistage génétique lié au BRCA ont créé de nouveaux défis pour les infirmières en oncologie œuvrant dans des contextes cliniques. Afin d'acquérir une meilleure compréhension des perspectives physiologique, pathophysiologique, comportementale et expérientielle, les infirmières se doivent de rechercher activement des opportunités de formation telles que la lecture d'articles de recherche, la participation à des conférences sur la génétique et une formation formelle en oncogénétique. La présente recension de la littérature permet de conclure que de nouvelles études de recherche sont requises en vue de mettre au point des recommandations fondées sur des données probantes relativement à l'utilisation des CO chez les femmes porteuses d'une mutation BRCA, de la HTS chez les porteuses qui n'ont pas subi de SOB et le risque de cancer du sein chez les porteuses dont l'organisme a été exposé à des 
rayonnements ionisants (Horsman, 2007; Willey et coll., 2007). Les résultats des études de Sivell et ses collègues (2007) ainsi que de Smith et ses collègues (2008) soulignent la nécessité de recherches continues sur les interventions infirmières relatives à l'évaluation psychologique des clientes traversant l'épreuve de consultation et de dépistage génétiques, en accordant une attention particulière à celles qui ont refusé le dépistage. Cette recension a également permis de dégager la nécessité d'effectuer des études de recherche afin d'évaluer la mise en place et l'évaluation d'aides à la décision pré- et post-consultation génétique pour la clientèle à risque élevé de cancer du sein dans le milieu de pratique clinique des soins infirmiers en oncologie.

\section{Références}

Barse, P.M. (2006). How to Perform a Genetic Assessment. In A.S. Tranin, A. Masny \& J. Jenkins (Eds.), Genetics in Oncology Practice: Cancer Risk Assessment (2nd ed., pp. 57-73). Pittsburgh, PA: Oncology Nursing Association.

Berliner, J.L., \& Fay, A.M. (2007). Risk assessment and genetic counseling for hereditary breast and ovarian cancer: Recommendations of the National Society of Genetic Counselors. The American Journal of Human Genetics, 16, 241-260.

Brown, K.L., Moglia, D.M., \& Grumet, S. (2007). Genetic counseling for breast cancer risk: General concepts, challenging themes and future directions. Breast Disease, 27, 69-96.

Carroll, J.C., Cremin, C., Allanson, J., Blaine, S.M., Dorman, H., Gibbons, C.A., et al. (2008). Hereditary breast and ovarian cancers. Official Publication of the College of Family Physicians of Canada, 54(12), 1691-1692.

Carter, R.F. (2001). BRCA1, BRCA2 and breast cancer: A concise clinical review. Clinical and Investigative Medicine, 24(3), 147-157.

Chapman, D.D. (2007). Cancer genetics. Seminars in Oncology Nursing, 23(1), 2-9.

Cuningham, K. (2008). Smoking and risk of breast cancer in carriers of mutations in BRCA1 or BRCA2 aged less than 50 years. Breast Cancer Research and Treatment, 109, 67-75.

Ensenauer, R.E., Michels, V.V., \& Reinke, S.S. (2005). Genetic testing: practical, ethical, and counseling considerations. Mayo Clinical Proceedings, 80(1), 63-73.

Evans, J.P., Skrzynia, C., Susswein, L., \& Harlan, M. (2005). Genetics and the young woman with breast cancer. Breast Diseases, 23, 17-29.

Fackenthal, J.D., \& Olopade, O.I. (2007). Breast cancer risk associated with BRCA1 and BRCA2 in diverse populations. Nat Rev Cancer, 7(12), 937-48.

Horsman, D., Wilson, B.J., Avard, D., Meschino, W.S., Sing, C.K., Plante, M., et al. On behalf of the National Hereditary Cancer Task Force. (2007). Clinical management recommendations for surveillance and risk-reduction strategies for hereditary breast and ovarian cancer among individuals carrying a deleterious BRCA1 or BRCA2 mutation. Journal of Obstetrics and Gynaecology Canada, 29(1), 45-60.

Kotsopoulos, J., Lubinski, J., Lynch, H. T., Klijn, J., Ghadirian, P., \& Neuhausen, S.L., et al. The Hereditary Breast Cancer Clinical Study Group. (2007). Age at first birth and the risk of breast cancer in BRCA1 and BRCA2 mutation carriers. Breast Cancer Research and Treatment, 105(2), 221-228.

Loescher, L.J., \& Whitesell, L. (2006). The Biology of Cancer. In A.S. Tranin, A. Masny \& J. Jenkins (Eds.), Genetics in oncology practice: Cancer risk assessment (2nd ed., pp. 23-55). Pittsburgh, PA: Oncology Nursing Association.

McGuire, V., John, E.M., Felberg, A., Haile, R.W., Boyd, N.F., \& Thomas, D.C., et al. (2006). No increased risk of breast cancer associated

\section{Conclusion}

Le diagnostic de cancer du sein héréditaire ou l'éventualité de ce dernier peut avoir une incidence sur de nombreux aspects du bienêtre de la personne concernée et des membres de sa famille. Le modèle HRTI offre aux infirmières cliniciennes en oncologie le cadre nécessaire à la compréhension de la biologie du cancer du sein héréditaire et la structure permettant d'obtenir une évaluation détaillée de l'histoire oncologique personnelle et familiale. Cette information peut être utilisée afin de personnaliser l'enseignement à la cliente et les soins de soutien. Ce modèle fournit donc aux infirmières en oncologie un excellent cadre facilitant la prestation de soins holistiques.

with alcohol consumption among carriers of BRCA1 and BRCA2 mutations ages <50 years. Cancer Epidemiology, Biomakers \& Prevention, 15, 1565-1567.

Mitchell, P.H., Gallucci, B., \& Fought, S.G. (1991). Perspectives on human response to health and illness. Nursing Outlook, 39(4), 154-157.

Narod, S.A., \& Foulkes, W.D. (2004). BRCA1 and BRCA2: 1994 and beyond. Nature Reviews Cancer, September(4), 665-676.

Nkondjock, A., \& Ghadirian, P. (2004). Epidemiology of breast cancer among BRCA mutation carriers: An overview. Cancer Letters, 205 , $1-8$.

Petrucelli, N., Daly, M.B., Culver, J.O., Feldman, G.L. (2007). BRCA1 and BRCA2 hereditary breast/ovarian cancer. Gene Reviews. Retrieved from http://www.ncbi.nlm.nih.gov/bookshelf/br.fcgi? book=gene\&part=brcal

Phelps, C., Wood, F., Bennett, P., Brain, K., \& Gray, J. (2007). Knowledge and expectations of women undergoing cancer genetic risk assessment: A qualitative analysis of free-test questionnaire comments. Journal of Genetic Counseling, 16(4), 505-514.

Ronckers, C.M., Erdmann, C.A., \& Land, C.E. (2005). Radiation and breast cancer: A review of current evidence. Breast Cancer Research, 7(1), 21-32.

Rosen, E.M., Fan, S., \& Isaacs, C. (2005). Review BRCA1 in hormonal carcinogenesis; basic and clinical research. Endocrine-Related Cancer, 12(3), 533-548.

Schlich-Bakker, K.J., ten Kroode, H.F.J., \& Ausems, M.G.E.M. (2006). A literature review of the psychological impact of genetic testing on breast cancer patients. Patient Education and Counseling, 62(1), 13-20.

Schwartz, M.D., Peshkin, B.N., Hughes, C., Main, D., Isaacs, C., \& Lerman. C. (2002). Impact of BRCA1/BRCA2 mutation testing on psychologic distress in a clinic-based sample. Journal of Clinical Oncology, 20(2), 514-520.

Sifri, R., Gangadharappa, S., \& Acheson, L.S. (2004). Identifying and testing for hereditary susceptibility to common cancers. A Cancer Journal for Clinicians, 54, 309-326.

Sivell, S., Iredale, R., Gray, J., \& Coles, B. (2007). Cancer genetics risk assessment for individuals at risk of familial breast cancer (Review). Cochrane Database of Systematic Reviews, (2), CD003721, 1-30.

Smith, A.W., Dougall, A.L., Posluszny, D.M., Somers, T.J., Rubinstein, W.S., \& Baum, A. (2008). Psychological distress and quality of life associated with genetic testing for breast cancer risk. Journal of Psychosocial Oncology, 17, 767-773.

Société canadienne du cancer/Institut national du cancer du Canada (2009). Statistiques canadiennes sur le cancer. ISSN 0835-2976.

Willey, S.C., \& Cocilovo, C. (2007). Screening and follow-up of the patient at high risk for breast cancer. Obstetrics and Gynecology, 110(6), 1404-1416.

Yoshida, K., \& Miki, Y. (2004). Role of BRCA1 and BRCA2 as regulators of DNA repair, transcription and cell cycle in response to DNA damage. Cancer Science, 95(11), 866-871. 